\title{
Compacting a Single Flow Diverter versus Overlapping Flow Diverters for Intracranial Aneurysms: A Computational Study
}

\author{
(D).J. Damiano, (D) V.M. Tutino, (D) N. Paliwal, DD. Ma, (D).M. Davies, (D)A.H. Siddiqui, and (D) H. Meng
}

\begin{abstract}
BACKGROUND AND PURPOSE: Locally compacting the mesh of a flow diverter by a dynamic push-pull technique can accelerate intracranial aneurysm healing. We asked how this deployment strategy compares with overlapping 2 flow diverters for aneurysmal flow reduction.
\end{abstract}

\begin{abstract}
MATERIALS AND METHODS: Using a high-fidelity virtual stent placement method, we simulated 3 flow-diverter strategies (single noncompacted, 2 overlapped, and single compacted) in 3 aneurysms (fusiform, large saccular, and medium saccular). Computational fluid dynamics analysis provided posttreatment hemodynamic parameters, including time-averaged inflow rate, aneurysm-averaged velocity, wall shear stress, total absolute circulation, and turnover time. We examined the relationship between the achieved degree of compaction and aneurysm orifice area.
\end{abstract}

RESULTS: Flow-diverter compaction resulted in a compaction coverage of $57 \%, 47 \%$, and $22 \%$ over the orifice of the fusiform, large, and medium saccular aneurysm, respectively. Compaction coverage increased linearly with orifice area. In the fusiform aneurysm, the single compacted flow diverter accomplished more aneurysmal flow reduction than the other 2 strategies, as indicated by all 5 hemodynamic parameters. In the 2 saccular aneurysms, the overlapped flow diverters achieved the most flow reduction, followed by the single compacted and the noncompacted flow diverter.

CONCLUSIONS: Compacting a single flow diverter can outperform overlapping 2 flow diverters in aneurysmal flow reduction, provided that the compaction produces a mesh denser than 2 overlapped flow diverters and this denser mesh covers a sufficient portion of the aneurysm orifice area, for which we suggest a minimum of $50 \%$. This strategy is most effective for aneurysms with large orifices, especially fusiform aneurysms.

ABBREVIATIONS: $C C=$ compaction coverage; $C F D=$ computational fluid dynamics; DPPT $=$ dynamic push-pull technique; FD = flow diverter; HiFiVS $=$ high-fidelity virtual stent placement; IA = intracranial aneurysm

Clow diversion is a minimally invasive, endovascular therapy for treating intracranial aneurysms (IAs). Flow diverters (FDs) are self-expandable, densely braided metallic stents that are deployed

Received May 23, 2016; accepted after revision October 27.

From the Department of Mechanical and Aerospace Engineering (R.J.D., N.P., D.M., H.M.), Toshiba Stroke and Vascular Research Center (R.J.D., V.M.T., N.P., D.M., J.M.D., A.H.S., H.M.), Department of Biomedical Engineering (V.M.T., H.M.), and Department of Neurosurgery (J.M.D., A.H.S., H.M.), University at Buffalo, State University of New York, Buffalo, New York.

This study was supported by the National Institutes of Health (R01 NS 091075) and Covidien (VTGCC053012-009).

Please address correspondence to Hui Meng, PhD, Department of Mechanical and Aerospace Engineering, University at Buffalo, State University of New York, Buffalo, NY 14260; e-mail: huimeng@buffalo.edu

- Indicates open access to non-subscribers at www.ajnr.org

三 Indicates article with supplemental on-line appendix.

$\checkmark$ Indicates article with supplemental on-line photo.

http://dx.doi.org/10.3174/ajnr.A5062 across the IA neck. Their low-porosity mesh redirects blood flow back into the parent vessel, decreasing flow into the aneurysm and thereby encouraging parent vessel reconstruction and aneurysm embolization. ${ }^{1}$ However, embolization does not occur immediately, leaving treated IAs at risk of rupture before complete occlusion is achieved. ${ }^{2}$ Treatment strategies aimed at reducing aneurysmal flow beyond that achievable by conventional FD deployment can decrease the time to occlusion and reduce rupture risk. ${ }^{3}$

One of these strategies is to overlap multiple FDs to increase mesh coverage over the aneurysm neck to accelerate IA occlusion. The strategy of overlapping FDs was used in 2 large clinical trials testing the safety and efficacy of the Pipeline Embolization Device (Covidien, Irvine, California) in treating unruptured aneurysms: the Pipeline Embolization Device for the Intracranial Treatment of Aneurysms ${ }^{4}$ and Pipeline for Uncoilable or Failed Aneurysms. ${ }^{5}$ Additional overlapping FDs were deployed to treat aneurysms at 
the discretion of the neurointerventionalists, and an analysis of treatments at the conclusions of the trials showed that the average number of FDs used per case was 1.52 and 3.1, respectively. These trials demonstrated 6-month occlusion rates of $93 \%$ and $73.6 \%$, respectively, supporting the efficacy of using overlapping FDs to treat IAs. Computational studies of sequentially overlapping stents for treating IAs have supported this concept, showing increased flow reduction with the increases in mesh coverage over the IA orifice. ${ }^{6}$ More recently, we simulated realistic deployment of the Pipeline Embolization Device and demonstrated that overlapping 2 devices reduced aneurysmal flow velocity 30\% more than deploying 1 device. ${ }^{7}$

However, negative clinical consequences of overlapping FDs have also been reported, including in-stent thrombosis, stenosis, and perforator occlusion. ${ }^{8,9}$ These complications are likely due to the increased metal surface area and lowered porosity due to additional FDs. ${ }^{8}$ It would be desirable to deploy a single FD but to achieve as much aneurysmal flow reduction as overlapping multiple FDs, to reduce the risk of these complications.

Most interesting, because its individual wires can slide over one another, an FD can generate spatially varying mesh density when deployed. This ability was demonstrated by several benchtop experiments that showed that when longitudinally compressed during deployment, an FD expands radially and its wires compact into a localized zone of higher mesh density. ${ }^{10,11}$ To use this property, we recently developed a novel deployment strategy to achieve local FD compaction for patient-specific IAs: the dynamic push-pull technique (DPPT). ${ }^{12}$ With this strategy, the mesh density of an FD can be increased over the IA orifice during deployment through synergistic push-and-pull maneuvers between the microcatheter and delivery wire. We demonstrated the use of the DPPT for patient-specific IAs in vitro by physically deploying FDs and compacting their meshes in several IA flow phantoms. ${ }^{13}$ We also showed that an FD compacted by using the DPPT reduced aneurysmal flow velocity $38 \%$ more than a noncompacted, uniformly deployed FD. ${ }^{14}$ A recent in vivo study of canine aneurysm models also found that compacted FDs resulted in more frequent occlusion than noncompacted FDs. ${ }^{15}$ We hypothesized that a single compacted FD could realize the benefits of overlapping multiple FDs without associated complications.

This study tested numerically whether compacting a single FD could be as effective as 2 overlapped FDs in aneurysmal flow reduction. To this end, we used a finite element method-based modeling technique - the high-fidelity virtual stenting (HiFiVS) ${ }^{12}$ method-to simulate various FD deployment strategies, including single noncompacted, 2 overlapped, and single compacted. Applying these modeling strategies, we virtually "treated" 3 representative patient-specific IAs: a fusiform, a large saccular, and a medium saccular aneurysm. We then performed computational fluid dynamics (CFD) analysis to assess hemodynamic modifications in each scenario and compared the resulting flow modifications by using 5 hemodynamic parameters.

\section{MATERIALS AND METHODS Creation of IA Models}

For this computational study, we chose 3 representative patientspecific IAs as test cases: a medium 9-mm fusiform basilar trunk
IA, a large 18-mm saccular internal carotid artery IA, and a medium 9.5-mm saccular internal carotid artery IA. They were all treated by FDs at Gates Vascular Institute (Buffalo, New York), and their pretreatment angiographic images were obtained at the time of treatment under institutional review board approval. Although all 3 cases were treated by FDs, the FD strategies tested in the current numeric study answered the question of the effectiveness of different competing treatment strategies and did not correspond to the actual treatments received by these patients.

To create the 3D IA models, we segmented their angiographic images by using the level set and marching cube methods in The Vascular Modeling Toolkit (www.vmtk.org). ${ }^{16}$ To make the FD deployment simulations computationally tractable, we assumed that the IA models were rigid. This assumption was reasonable because FDs exhibit lower radial forces ${ }^{1}$ than closed-pore stents and thus have less effect on vessel morphology.

\section{FD Size Selection}

We selected the FD size for each IA by following standard clinical procedures. ${ }^{17}$ The nominal diameter of an FD was chosen to approximate the size of the recipient parent vessel in each IA, and the nominal length was chosen to be at least $6 \mathrm{~mm}$ longer than the length of the IA neck. This process gave rise to $3 \mathrm{FD}$ specifications for each IA: FDs measuring at least $5 \times 13,4.25 \times 12$, and $3.25 \times$ $10 \mathrm{~mm}$ in the fusiform, large, and medium IAs, respectively.

\section{Modeling the Deployment Strategies of Noncompacting and Overlapping FDs with HiFivs}

The HiFiVS ${ }^{12}$ method was used to virtually deploy FDs in this study. As previously described, HiFiVS is a finite element method-based FD simulation technique that models several components of the FD delivery system, including the crimper, microcatheter, distal coil, and proximal pusher. All deployment mechanics and critical steps that affect the final configurations of FDs were simulated, including crimping, delivery, release, and expansion.

The HiFiVS method was rigorously validated in our previous experimental studies. ${ }^{12,13}$ Side-by-side comparisons between experimental deployment of FDs in transparent IA phantoms and in silico deployment in identical IA models showed excellent agreement, as demonstrated in Fig 6 of Ma et al (2013). ${ }^{12}$ Local mesh characteristics of compacted FDs deployed by HiFiVS matched those deployed experimentally by the DPPT, as demonstrated in Fig 2 of Ma et al (2014). ${ }^{13}$

In the current study, we modeled the FDs after the Pipeline Embolization Device. The wire braiding pattern for each FD was generated in Matlab (MathWorks, Natick, Massachusetts) on the basis of mathematic description. ${ }^{18}$ The FD deployment procedure was simulated by using the finite element solver Abaqus/Explicit 6.13 (SIMULIA; 3DS, Waltham, Massachusetts).

To virtually deploy an FD without compaction, we retracted the microcatheter proximally while holding the distal coil still to produce uniform mesh density over the IA orifice. To simulate the deployment of 2 overlapped FDs, we ran 2 independent HiFiVS simulations consecutively. ${ }^{7}$ Following the deployment of the first FD in each IA $(5 \times 20,4.5 \times 20$, and $3.25 \times 14 \mathrm{~mm}$ for the fusiform, large, and medium IAs, respectively), a second FD mea- 
suring $5 \times 20,4.5 \times 14$, and $3.25 \times 12 \mathrm{~mm}$ was coaxially deployed in the fusiform, large, and medium IAs, respectively.

\section{Modeling the Deployment Strategy of Compacting a Single FD with HiFivs}

The previously described modeling technique of emulating the DPPT $^{12,13}$ was used in the current study to compact a single FD. This consisted of retracting the microcatheter proximally as in regular noncompacting deployment to unsheathe the FD, while advancing the proximal pusher distally to generate dense FD mesh over the IA orifice.

To generate the most flow diversion in each IA, we maximized the FD compaction during deployment by maneuvering the movement of the microcatheter and proximal pusher. The simulation procedure was stopped if the FD started to migrate distally or the wires of the FD began to unwind or tangle. Using this procedure, we maximally compacted FDs measuring $5 \times 20$, $4.5 \times 20$, and $3.25 \times 14 \mathrm{~mm}$ in the fusiform, large, and medium IAs, respectively.

\section{Quantification of FD Mesh Characteristics}

The amount of aneurysmal flow reduction achievable by an FD in a patient-specific IA is affected by its mesh porosity and pore density in its fully deployed state. ${ }^{1}$ An ideal FD has low porosity and high pore density to maximize flow diversion ${ }^{1}$ : Porosity represents the amount of empty space created by the mesh pores of the FD, and pore density, the number of pores per unit area. ${ }^{19} \mathrm{We}$ quantified these parameters near the IA orifice along the length of a deployed FD in 5 consecutive zones: proximal vessel, proximal transition, middle, distal transition, and distal vessel, as defined previously on the basis of an FD compacted via the DPPT. ${ }^{13}$ The proximal/distal vessel zones were the portions of the FD constrained in the parent vessel. The FD mesh in the middle zone (ie, over the IA orifice) was compacted because the goal of the DPPT is to increase mesh density over the IA orifice. ${ }^{13}$ The remainder of the IA orifice not covered by the middle zone was covered by the proximal/distal transition zones. Even though FDs deployed without compaction presented more or less uniform meshes, to compare porosity and pore density between different strategies, we analyzed all FDs in these 5 zones.

\section{Quantification of FD Mesh Compaction}

When an FD is axially compressed, 2 things happen simultaneously: The woven wires of the FD compact together and the FD construct expands radially. With FD deployment via the DPPT, the aim is to create local mesh compaction over the IA orifice to block flow into the IA. ${ }^{13}$ While the FD wires compact together, the IA orifice provides space to allow the FD construct to expand during compaction. Clearly the size of the orifice limits the degree to which an FD can be compacted and thus the effectiveness of the FD compaction strategy at improving aneurysmal flow reduction. Therefore, we investigated the relationship between the IA orifice area and the achievable compaction. This information could help clinicians determine the types of IA geometry for which the FD compaction strategy is most effective.

To that end, we measured IA orifices on the plane that separates the IA sac from the parent vessel for the saccular IAs and on the plane that separates the largest bulge from the parent vessel for the fusiform IA. Once the orifice was identified, we quantified the degree of achievable FD compaction by a new parameter, the compaction coverage (CC), as defined in Equation 1. Here, compaction coverage represents the percentage of the IA orifice covered by the middle zone of the compacted FD.

$$
\text { CC }(\%)=\frac{\text { Compaction Zone Area }}{\text { IA Orifice Area }}
$$

\section{CFD Simulations}

Pre- and posttreatment hemodynamics in all IA models were simulated by using CFD in Star-CCM+ v.10 (CD-adapco, Melville, New York). Blood flow was assumed to be pulsatile, laminar, incompressible, and Newtonian (density $=1056 \mathrm{~kg} / \mathrm{m}^{3}$, dynamic viscosity $=3.5 \mathrm{cP}$ ). A patient-specific, pulsatile velocity waveform was prescribed at each inlet, having a mean velocity matching typical flow rates at each IA location. A traction-free boundary condition was specified at each outlet. ${ }^{7}$ To solve for the aneurysmal flow fields, we spatially discretized each IA into unstructured grids by using polyhedral cells. ${ }^{7}$ Additional details of the CFD meshing parameters and the results of a mesh independence study are included in the On-line Appendix. Three cardiac cycles were simulated to ensure numeric stability of the flow solutions in all IA models. All hemodynamic parameters presented were time averages over the third cycle.

To compare aneurysmal flow modifications by the 3 FD strategies, we assessed posttreatment changes of 5 hemodynamic parameters: inflow rate, aneurysm-averaged velocity, wall shear stress, total absolute circulation, and turnover time. Previous studies reported that FDs reduced inflow rate, velocity, and wall shear stress and increased turnover time and that the amount of change correlated with IA occlusion rates. ${ }^{20,21}$ In addition, we also examined posttreatment changes in the total absolute hydrodynamic circulation. This new parameter is a modified version of hydrodynamic circulation that was used previously to compare aneurysmal flow changes by several custom-designed FDs. ${ }^{19}$ By definition, hydrodynamic circulation is an integration of vorticity flux over a region. Because the positive and negative vortex subregions could cancel out and give zero or small total circulation in this region, the use of hydrodynamic circulation could mask the true amount of rotational flow activity in this region. To capture the total amount of rotational flow activity of both positive and negative vortices in an aneurysm, we defined the total absolute circulation $\left(\Gamma_{A}\right)$ as the integration of the absolute value of vorticity flux over the IA midplane as shown in Equation 2:

$$
\Gamma_{A}=\iint|\vec{\omega} \cdot \hat{n}| d A
$$

where $\vec{\omega}$ is the local time-averaged vorticity, $\vec{\omega} \cdot \hat{n}$ is its component normal to the IA midplane, and the integration is over the entire midplane. The IA midplane is formed by 2 vectors: 1 ) the vector pointing from the centroid of the IA orifice to the centroid of the IA sac, and 2) the vector pointing from the centroid of the crosssection of the parent vessel immediately proximal to the IA to the centroid of the parent vessel cross-section immediately distal. We 


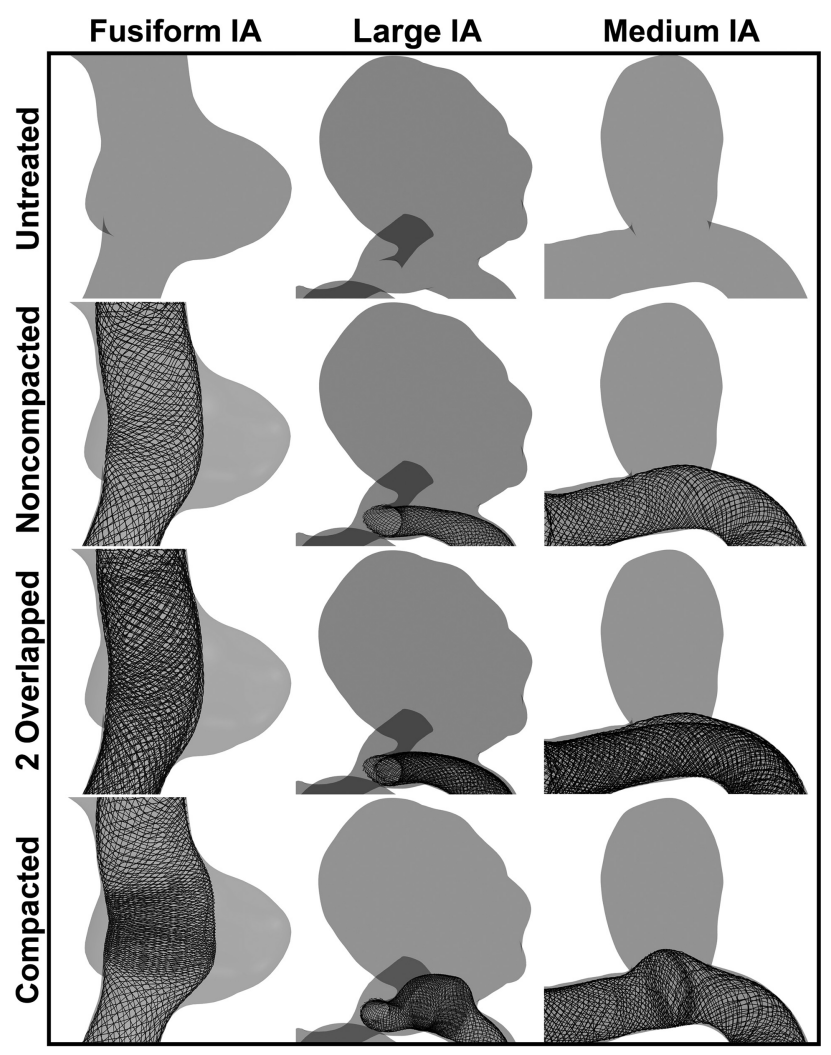

FIG 1. FD deployment results in all 3 IAs.

define the IA sac as the volume enclosed by the wall of the IA and the IA orifice.

\section{RESULTS}

\section{Compacting a Single FD Achieved Similar Porosity and Pore Density as Overlapping FDs}

Figure 1 shows the geometry of the 3 IA models and the FDs virtually deployed by the $3 \mathrm{FD}$ treatment strategies in each IA. The fusiform IA (on the basilar artery) involves the entire circumference of the parent vessel, with the largest bulge protruding to the right (left column of Fig 1). The 2 saccular IAs both occur sidewall on the internal carotid artery. After treatment, the mesh density appears rather uniform in all 3 IA models for the noncompacted FD and overlapped FDs. The compacted FD, on the other hand, shows highly variable mesh density in all 3 cases, bulging into the IA sac and compacting in a distinct zone of higher mesh density. The middle zone is largest in the fusiform IA and decreases in the large and medium IAs. The remaining zones of the compacted FD are less dense in comparison with the middle zone in all 3 IAs.

Figure 2 shows the quantification of mesh variations in terms of porosity and pore density of all virtually deployed FDs. The 5 mesh zones for calculating these quantities are illustrated in Fig $2 A$, with the fusiform IA as an example, wherein the middle zone (M) of the compacted FD has much higher mesh density over other zones. Figure $2 B,-C$ shows porosity and pore density, respectively, plotted over the consecutive mesh zones for all FD strategies. Overall, the single noncompacted FDs have the highest porosity and lowest pore density, while the overlapped FDs have the lowest porosity and highest pore density. The compacted FDs show large variations in both porosity and pore density along their lengths. They are generally similar to those in the single noncompacted FDs, but in the middle zone, they have a large jump, approaching or exceeding the overlapped FDs, except for 2 cases.

\section{Compaction Coverage Increased Linearly with IA Orifice Area}

To explore the relationship between the maximally achievable FD compaction and IA geometry, we quantified the IA orifice area, the area of compaction, and the compaction coverage. Figure 3 shows the FD mesh at the orifices of the 3 IAs and the CC plotted against the orifice area. The fusiform IA orifice had the largest coverage by the middle zone (ie, the compacted portion), and the medium IA orifice had the smallest (Fig 3A). Conversely, the medium IA orifice had the largest coverage by the transition zones (ie, the noncompacted portions), and the fusiform IA orifice had the smallest. The orifice areas for the fusiform, large, and medium IAs were $34.5,30.0$, and $14.7 \mathrm{~mm}^{2}$, respectively, while the corresponding CCs were $57 \%, 47 \%$, and $22 \%$, respectively. Figure $3 B$ demonstrates a strong linear correlation between the CC and IA orifice area $\left(R^{2}=0.9965\right)$. In both saccular IAs, CC was below $50 \%$, meaning less than half of the IA orifice was covered by the middle zone.

\section{Compacting a Single FD Outperformed Overlapping 2 FDs} in Aneurysmal Flow Reduction in the Fusiform IA

We performed CFD simulations for all 12 IA models shown in Fig 1 , including the 3 untreated IAs, and posttreatment hemodynamics by using all 3 treatment strategies in each IA. Figure 4 shows the volume-rendered time-averaged velocity magnitude in all 12 IA models. In addition to the velocity magnitude (rendered in color), streamlines (rendered in black) are plotted to visualize the flow modifications by each FD strategy. In the fusiform IA, a strong vortex was formed in the large bulge, and it was diminished by the FDs to various extents, with the compacted FD delivering the most reduction. In the large and medium saccular IAs, an inflow jet entered the IA sac distally and circulated throughout the IA sac before exiting proximally. In the large IA, the size and velocity of the inflow jet was reduced most by the compacted FD, followed by the overlapped FDs and then the noncompacted FD. On the other hand, in the medium IA, the overlapped FDs reduced the size and velocity of the inflow jet the most. The compacted FD appeared to disrupt the inflow jet as much as, but no more than, the noncompacted FD in the medium IA.

To quantify the different effects of flow reduction by the $3 \mathrm{FD}$ strategies, we calculated posttreatment modifications in the timeaveraged inflow rate, aneurysm-averaged velocity, wall shear stress, total absolute circulation, and turnover time, all shown in Fig 5. Regardless of the deployment strategy, FDs reduced intraaneurysmal flow in all 3 IAs, as evidenced by the decrease in inflow rate, aneurysm-averaged velocity, wall shear stress, and total absolute circulation and an increase in turnover time from the untreated IAs. In the fusiform IA, the compacted FD outperformed the overlapped FDs in all 5 hemodynamic parameters. In contrast, the overlapped FDs outperformed the compacted FD in the large and medium IAs, except for total absolute circulation in the large IA, where the compacted FD performed best. 


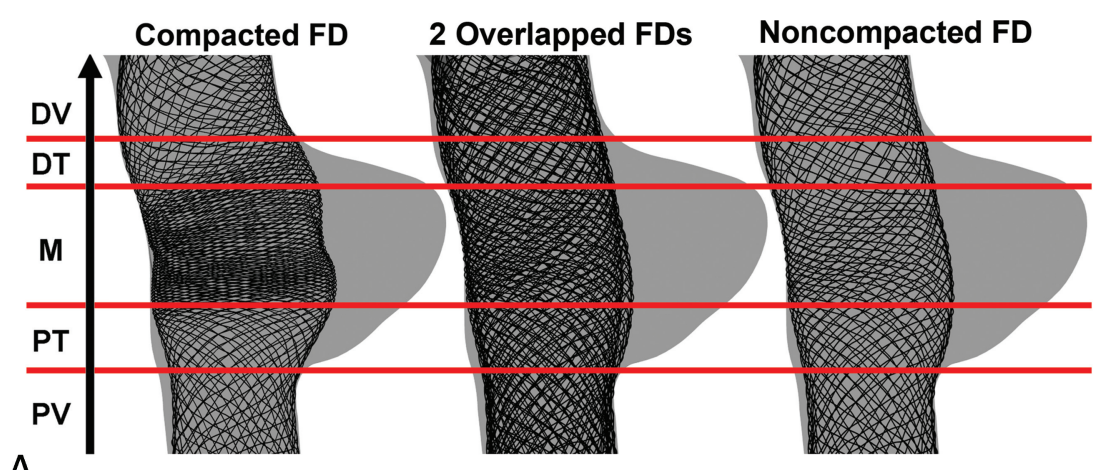

A
Fusiform IA
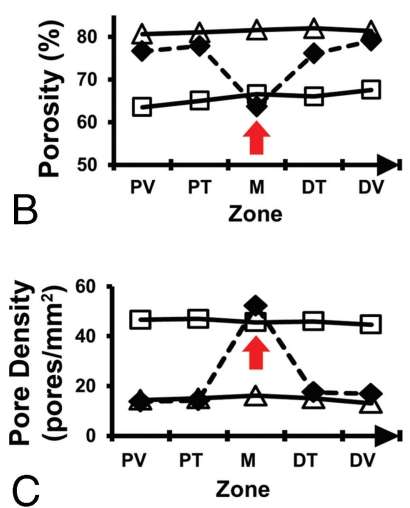

$\triangle$ Noncompacted FD $\boxminus 2$ Overlapped FDs
Large IA
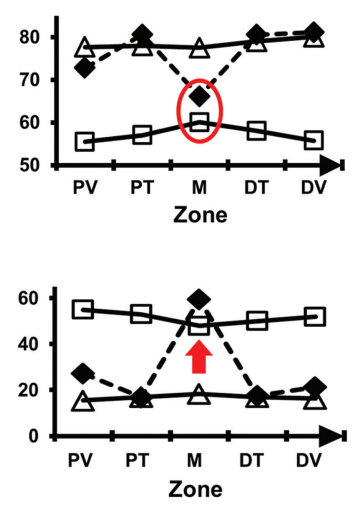

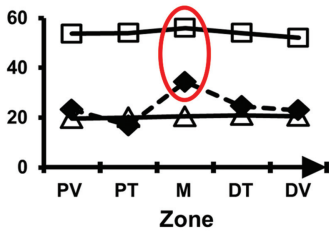

Compacted FD

FIG 2. Porosity and pore density distributions of deployed FDs resulting from all 3 deployment methods in the 3 IAs. A, Deployed FD meshes in the fusiform IA and demarcations of FD zones used for porosity and pore density calculations: proximal vessel (PV), proximal transition (PT), middle (M), distal transition (DT), and distal vessel (DV). These zones are defined on the basis of the compacted FD but applied to all 3 deployment strategies for calculation of porosity and pore density. B, Porosity distribution. C, Pore density distribution. The arrows indicate middle zones in which the compacted FD has lower porosity or higher pore density than the overlapped FDs, whereas the ovals indicate middle zones in which it does not.

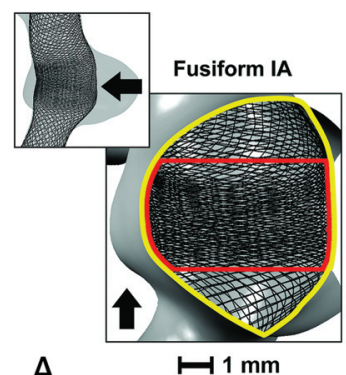

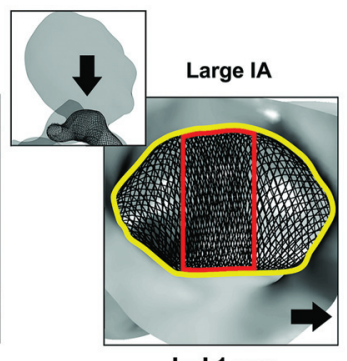

$\longmapsto 1 \mathrm{~mm}$

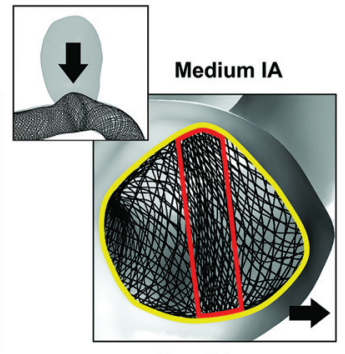

$\longmapsto 1 \mathrm{~mm}$

A

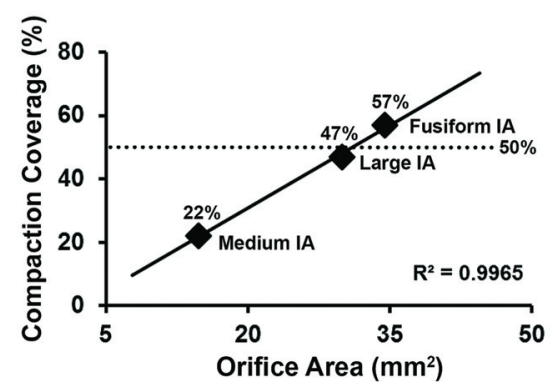

B

FIG 3. FD mesh compaction achieved in the 3 IAs. A, Compacted meshes at the IA orifice. Orifice areas are highlighted by the circular regions, and the compaction zones, by the rectangular regions. The arrows in the smaller schematics of each IA indicate viewing directions at each IA orifice. The arrows in the larger schematics of each IA orifice indicate the flow direction. Scale bars below each IA orifice indicate distances of $1 \mathrm{~mm}$. B, Plot of compaction coverage (percentage) versus IA orifice area, showing a strong linear relationship.

\section{DISCUSSION}

In this computational study, we tested whether a single compacted FD could outperform 2 overlapped FDs in IA flow reduction. Our results suggest that this goal is achievable provided that the compaction of the single FD can achieve 2 conditions: 1) It produces a compacted mesh in the middle zone that is denser than 2 overlapped FDs, as judged by both porosity and pore density; and 2) this denser mesh covers a sufficient portion of the IA orifice area, of which we suggest a minimum of $50 \%$.

In the fusiform IA model, compacting a single FD achieved both of these conditions. Not surprising, the compacted FD outperformed the 2 overlapped FDs in all 5 hemodynamic parameters. In the 2 saccular IAs, however, compacting a single FD did not achieve both compaction conditions above. In the large saccular IA, the porosity did not get down to the level of the 2 overlapped FDs (Fig 2B), while in the medium saccular IA, the pore density did not reach that of the overlapped FDs (Fig 2C) and neither IA allowed CC of $>50 \%$. We believe that this inadequate compaction was responsible for the flow simulation results in the 2 saccular IAs - that is, the compacted FD did not outperform the overlapped FDs in aneurysmal flow reduction. We further found that the degree to which an FD can be compacted and thus the ability to achieve both mesh conditions when compacting an FD are linearly related to the area of the aneurysm orifice. An FD can be compacted to a larger degree over an IA with a larger orifice because a large orifice provides more space for the FD to expand. This radial expansion occurs to accommodate the compaction of the woven wires of the FD as the FD is axially compressed. Therefore, the salient finding of this study is that compacting a single FD can outperform overlapping 2 FDs in aneurysms with large orifices, especially fusiform IAs.

We believe that compacting a single FD could be a superior strategy to overlapping multiple FDs, from both a biologic and economic standpoint. One concern with overlapping multiple FDs is that it increases the mesh density in the parent vessel, thereby increasing the 


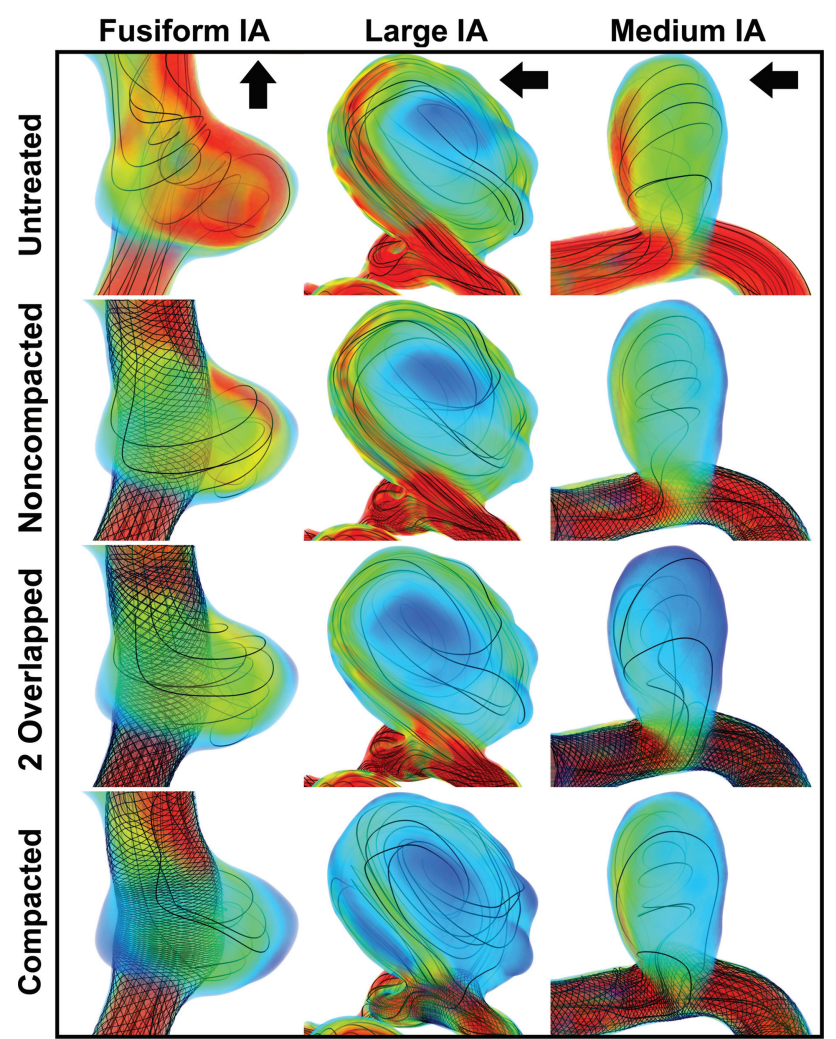

Time-Averaged Velocity Magnitude $(\mathrm{m} / \mathrm{s})$

0.0

0.1

0.2

0.3

FIG 4. Time-averaged, volume-rendered velocity magnitude in all 3 IAs for each FD strategy. Streamlines are plotted to enhance visualization of flow modifications due to each FD strategy. The arrows indicate the flow direction for each untreated IA.

chance of perforator occlusion. ${ }^{8}$ Overlapping multiple metal devices also increases the risk for in-stent thrombosis and stenosis. ${ }^{9}$ In contrast, the strategy of compacting a single FD increases the mesh density only over the IA orifice, but not in the parent vessel, thereby minimizing the risk of occluding nearby perforators. In addition, this strategy uses a single device, thereby minimizing the amount of thrombogenic metal and eliminating the costs and procedural risks associated with additional devices.

The goal of compacting an FD is to increase mesh density over the IA orifice beyond that achievable by not compacting an FD, in the hope of accelerating IA occlusion. However, failing to achieve adequate mesh compaction may render the compacted FD no more effective at aneurysmal flow reduction than a noncompacted FD. This result is because the less area of an FD that is compacted over the IA orifice, the more area of the orifice that is left at a lower mesh density. In other words, the smaller the middle zone, the larger is the transition zones. Benchtop experiments have shown that mesh transition zones are unavoidable when compacting an FD. ${ }^{10}$ In addition, the experiments showed that the transition zones were always less dense than the middle zone; this feature could compromise the ability of the FD mesh to divert aneurysm inflow. Our study supports this observation. We found that the mesh transition zones of the compacted FDs were approximately as dense as those of the noncompacted FDs in all 3 IAs (Fig 2B, $-C$ ). The implications of this result in our study were
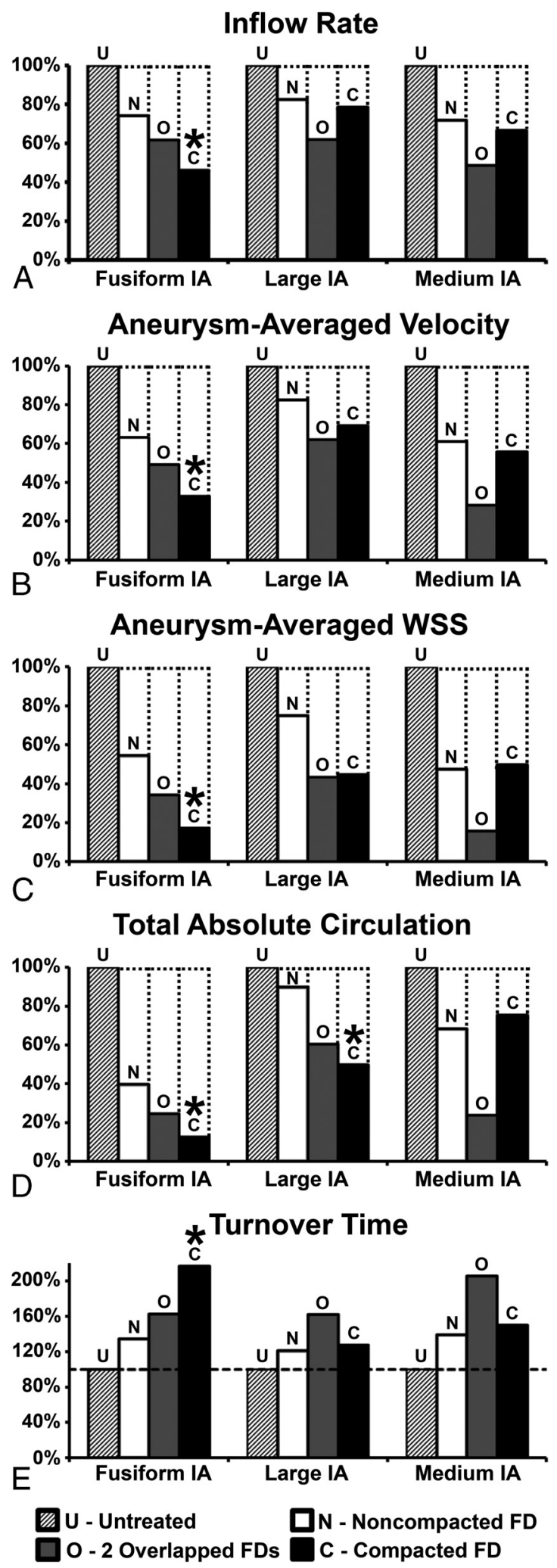

FIG 5. Changes in time-averaged hemodynamic parameters by FDs in each IA, relative to the untreated IAs. $A$, Inflow rate at the IA orifice. $B$ Aneurysm-averaged velocity in the IA sac. $C$, Aneurysm-averaged wall shear stress on the wall of the IA sac. D, Total absolute circulation at the IA midplane. E, Turnover time in the IA sac. Changes in hemodynamic parameters by FDs are reported as percentages of the values in the untreated IAs. The asterisks indicate instances in which the compacted FD outperformed the overlapped FDs. 
most revealing when considering the medium IA, which has the smallest orifice area of the 3 IA cases. In this aneurysm, the compacted FD disrupted the IA inflow jet as much as but no more than the noncompacted FD, and the jet passed through the distal transition zone of the FD (Fig 4). We could not further compact the FD due to the small orifice ( $<50 \%$ CC). Consequently, most of the orifice was covered by the lower mesh density transition zones. Thus, inadequate compaction of an FD leaves the orifice covered by lower density mesh, thereby delaying or preventing thrombosis due to insufficient flow diversion. On the basis of our results, we suggest ensuring that at least $50 \%$ of the IA orifice is covered by the higher density middle zone when compacting an FD.

On the other hand, excessive compaction of an FD could also cause complications, for example, causing the FD to prolapse into the aneurysm or migrate along the parent vessel. Gentric et al, ${ }^{15}$ in an attempt to treat several canine aneurysm models by compacting single FDs in vivo, experienced excessive compaction, which caused 4 FDs to prolapse into the aneurysm sac during deployment and 1 to migrate distally along the parent vessel following deployment. In the current in silico study, we also saw that if too much force was applied to the proximal pusher during compaction, the FD would migrate along the parent vessel. Consequently, we had to rerun the simulation with less force applied to ensure that the FD was anchored properly in the parent vessel. In the clinical setting, however, it would be extremely hard to salvage a prolapsed or migrated FD after deployment. Therefore, extra precaution is required to avoid excessively compacting an FD and causing FD prolapse or migration during the intervention.

Our study shows that no FD deployment strategy, including compacting FDs, is optimal for all IAs. We believe that IA geometry is the most important determining factor for the success of any flow-diversion strategy. Other factors also come into play as well. For example, the type and dimensions of the FD devices will determine the deployment process and the resulting mesh characteristics (eg, porosity and pore density). These, in turn, will determine the flow-diversion effect of the FD strategy (as demonstrated by Mut et $\mathrm{al}^{22}$ ). Furthermore, the success of the FD treatment also depends on the experience level of the neurointerventionalist. A steep learning curve is well-recognized in performing FD deployments, and the deployment procedure requires substantial technical ability, ${ }^{23}$ especially for complex deployment techniques such as compacting or overlapping FDs.

\section{CONCLUSIONS}

We investigated whether compacting a single FD could outperform overlapping 2 FDs in aneurysmal flow reduction in 3 patient-specific IAs. We found that compacting a single FD can be as effective as overlapping 2 FDs in aneurysmal flow reduction, provided that the compaction of the single FD can produce a dense mesh in the middle zone that is denser than 2 overlapped FDs and this denser mesh covers a sufficient portion of the IA orifice area, of which we suggest a minimum of $50 \%$. FD compaction is most suitable for IAs with large orifice areas, especially fusiform IAs.

\section{ACKNOWLEDGMENTS}

The authors thank the Center for Computational Research at the University at Buffalo for providing computational resources.

Disclosures: Adnan H. Siddiqui-UNRELATED: Board Membership: Intersocietal Accreditation Committee; Consultancy: Codman \& Shurtleff, Medtronic, GuidePoint Global Consulting, Penumbra, Stryker, MicroVention, W.L. Gore \& Associates, Three Rivers Medical Center, Corindus Vascular Robotics, Amnis Therapeutics, CereVasc, Pulsar Vascular, The Stroke Project, Cerebrotech Medical Systems, Rapid Medical, Lazarus (acquired by Medtronic), Medina Medical (acquired by Medtronic), Reverse Medical (acquired by Medtronic), Covidien (acquired by Medtronic), Neuroavi, Silk Road Medical, Rebound Medical; Consulting Fee or Honorarium: Medtronic, MicroVention; Stock/Stock Options: StimSox, Valor Medical, Neuro Technology Investors, Cardinal Health, Medina Medical, Buffalo Technology Partners, International Medical Distribution Partners; Other: Principal Investigators/National Steering Committees for various trials, Comments: the Penumbra 3D Separator Trial, the Covidien (Now Medtronic) Solitaire With the Intention For Thrombectomy as PRIMary Endovascular Treatment Trial and SWIFT DIRECT Trials, the MicroVention FRED Trial and CONFIDENCE Study, the LARGE Trial, POSITIVE Trial, Penumbra COMPASS Trial, and Penumbra INVEST Trial. Hui Meng—RELATED: Grant: National Institutes of Health*; UNRELATED: Consultancy: National Institutes of Health study sections*; Grants/ Grants Pending: Brain Aneurysm Foundation*; Payment for Lectures Including Service on Speakers Bureaus: University of California, San Francisco*; Patents (Planned, Pending, or Issued): University at Buffalo.* *Money paid to the institution.

\section{REFERENCES}

1. Sadasivan C, Cesar L, Seong J, et al. An original flow diversion device for the treatment of intracranial aneurysms: evaluation in the rabbit elastase-induced model. Stroke 2009;40:952-58 CrossRef Medline

2. Kulcsár Z, Houdart E, Bonafé A, et al. Intra-aneurysmal thrombosis as a possible cause of delayed aneurysm rupture after flow-diversion treatment. AJNR Am J Neuroradiol 2011;32:20-25 CrossRef Medline

3. Szikora I, Berentei Z, Kulcsar Z, et al. Treatment of intracranial aneurysms by functional reconstruction of the parent artery: the $\mathrm{Bu}$ dapest experience with the Pipeline Embolization Device. AJNR Am J Neuroradiol 2010;31:1139-47 CrossRef Medline

4. Nelson PK, Lylyk P, Szikora I, et al. The Pipeline embolization device for the intracranial treatment of aneurysms trial. AJNR Am J Neuroradiol 2011;32:34-40 CrossRef Medline

5. Becske T, Kallmes DF, Saatci I, et al. Pipeline for uncoilable or failed aneurysms: results from a multicenter clinical trial. Radiology 2013; 267:858-68 CrossRef Medline

6. Kim M, Levy EI, Meng H, et al. Quantification of hemodynamic changes induced by virtual placement of multiple stents across a wide-necked basilar trunk aneurysm. Neurosurgery 2007;61:130512; discussion 1312-13 CrossRef Medline

7. Damiano RJ, Ma D, Xiang J, et al. Finite element modeling of endovascular coiling and flow diversion enables hemodynamic prediction of complex treatment strategies for intracranial aneurysm. J Biomech 2015;48:3332-40 CrossRef Medline

8. Tan LA, Keigher KM, Munich SA, et al. Thromboembolic complications with Pipeline Embolization Device placement: impact of procedure time, number of stents and pre-procedure P2Y12 reaction unit (PRU) value. J Neurointerv Surg 2015;7:217-21 CrossRef Medline

9. Tse MM, Yan B, Dowling RJ, et al. Current status of Pipeline embolization device in the treatment of intracranial aneurysms: a review. World Neurosurg 2013;80:829-35 CrossRef Medline

10. Makoyeva A, Bing F, Darsaut TE, et al. The varying porosity of braided self-expanding stents and flow diverters: an experimental study. AJNR Am J Neuroradiol 2013;34:596-602 CrossRef Medline

11. Shapiro M, Raz E, Becske T, et al. Variable porosity of the Pipeline embolization device in straight and curved vessels: a guide for optimal deployment strategy. AJNR Am J Neuroradiol 2014;35:727-33 CrossRef Medline

12. Ma D, Dumont TM, Kosukegawa $H$, et al. High fidelity virtual stent- 
ing (HiFiVS) for intracranial aneurysm flow diversion: in vitro and in silico. Ann Biomed Eng 2013;41:2143-56 CrossRef Medline

13. $\mathrm{MaD}$, Xiang J, Choi $\mathrm{H}$, et al. Enhanced aneurysmal flow diversion using a dynamic push-pull technique: an experimental and modeling study. AJNR Am J Neuroradiol 2014;35:1779-85 CrossRef Medline

14. Xiang J, Ma D, Snyder KV, et al. Increasing flow diversion for cerebral aneurysm treatment using a single flow diverter. Neurosurgery 2014;75:286-94; discussion 294 CrossRef Medline

15. Gentric JC, Salazkin I, Gevry G, et al. Compaction of flow diverters improves occlusion of experimental wide-necked aneurysms. J Neurointerv Surg 2016;8:1072-77 CrossRef Medline

16. Antiga L, Piccinelli M, Botti L, et al. An image-based modeling framework for patient-specific computational hemodynamics. Med Biol Eng Comput 2008;46:1097-112 CrossRef Medline

17. Eller JL, Dumont TM, Sorkin GC, et al. The Pipeline embolization device for treatment of intracranial aneurysms. Expert Rev Med Devices 2014;11:137-50 CrossRef Medline

18. Ma D, Dargush GF, Natarajan SK, et al. Computer modeling of deployment and mechanical expansion of neurovascular flow di- verter in patient-specific intracranial aneurysms. J Biomech 2012; 45:2256-63 CrossRef Medline

19. Lieber BB, Sadasivan C. Endoluminal scaffolds for vascular reconstruction and exclusion of aneurysms from the cerebral circulation. Stroke 2010;41:S21-25 CrossRef Medline

20. Xiang J, Damiano RJ, Lin N, et al. High-fidelity virtual stenting: modeling of flow diverter deployment for hemodynamic characterization of complex intracranial aneurysms. J Neurosurg 2015;123: 832-40 CrossRef Medline

21. Mut F, Raschi M, Scrivano E, et al. Association between hemodynamic conditions and occlusion times after flow diversion in cerebral aneurysms. J Neurointerv Surg 2015;7:286-90 CrossRef Medline

22. Mut F, Cebral JR. Effects of flow-diverting device oversizing on hemodynamics alteration in cerebral aneurysms. AJNR Am J Neuroradiol 2012;33:2010-16 CrossRef Medline

23. Jabbour P, Chalouhi N, Tjoumakaris S, et al. The Pipeline Embolization Device: learning curve and predictors of complications and aneurysm obliteration. Neurosurgery 2013;73:113-20; discussion 120 CrossRef Medline 